\title{
Multi-plane phase retrieval methods to recover free- aberrations object complex field via SLM
}

\section{Métodos iterativos multi-plano para la recuperación del campo complejo del objeto libre de aberraciones usando un SLM}

\author{
R. Restrepo ${ }^{1 *}$, T.Belenguer ${ }^{2}$ \\ 1. Applied Optics Group, Universidad EAFIT, Carrera 49\#7 Sur-50, Medellín, Colombia \\ 2. Instituto Nacional de Técnica Aeroespacial (INTA), Área de Instrumentación Óptica Espacial, Carretera de \\ Ajalvir, Km. 4, Torrejón de Ardoz, Madrid, Spain, 28850 \\ (*) E-mail: rrestre6@eafit.edu.co \\ S: miembro de SEDOPTICA / SEDOPTICA member \\ Recibido: 02/12/2016 Accepted: 17/10/2017 \\ DOI: 10.7149/OPA.50.4.49066
}

\begin{abstract}
:
This paper explains two iterative phase recovery methods where the goal of each one is different. The Phase Diversity method is used to recover the wavefront aberrations in optical incoherent imaging systems when an extended object is illuminated. The purpose of the Multi-plane Phase Retrieval method is recovering the object complex field seen from the image plane, therefore the object has to be illuminated with coherent light. Both methods are simultaneously used to pick out the aberrations and free aberrations object phase. The use of both methods is proposed as a methodology for the integration of optical instruments.
\end{abstract}

Key words: phase retrieval methods, optical aberrations.

\section{RESUMEN:}

Este artículo explica dos métodos iterativos para la recuperación de la fase, donde el objetivo de cada método es diferente. El método de Diversidad de Fase se usa para recuperar las aberraciones ópticas de un sistema formador de imagen usando iluminación incoherente y un objeto extendido. El propósito del método Multi-plano Iterativo de Recuperación de Fase es recuperar el campo complejo del objeto y por tanto la iluminación debe ser coherente. Ambos métodos se usan simultáneamente para obtener tanto las aberraciones como la fase del objeto libre de aberraciones. El uso de ambos métodos se propone como una metodología para la integración de instrumentos ópticos.

Palabras clave: métodos de recuperación de fase iterativos, aberraciones ópticas

\section{REFERENCES AND LINKS / REFERENCIAS Y ENLACES}

[1] J. A. Bonet, High Spatial Resolution Imaging Solar Physics. Astrophysics and Space Science Library (1999).

[2] A. Anand, B. Javidi, "Three-dimensional microscopy with single-beam wavefront sensing and reconstruction from speckle fields," Optics letters, 35:766 (2010).

[3] K. B. Doyle, V. L. Genberg, G. J. Michels, Integrated Optomechanical Analysis. SPIE Press, 1st edition, (2002).

[4] G. Ming Dai, Wavefront Optics for Vision Correction. SPIE Press, 1st edition (2008).

[5] S. Fürhapter, A. Jesacher, S. Bernet, M. Ritsch-Marte, "Spiral interferometry," Optics Letters, 30:1953 (2005).

[6] R. W. Gerchgerg, W. O. Saxton, "A practical Algorithm for the Determination of Phase from Image and Diffraction Plane Pictures," Optik, 35:237 (1972). 
[7] F. Roddier, C. Roddier, "Wavefront reconstruction using iterative Fourier transforms," Applied Optics, 30:1325 (1991).

[8] C. Torti, S. Gruppetta, L. Diaz-Santana, "Wavefront curvature sensing for the human eye," Journal of Modern Optics, 55:691 (2008).

[9] V. Yu. Ianov, V. p. Sivokon, M. A. Vorontsov, "Phase retrieval from a set of intensity measurements: theory and experiment," Journal of the Optical Society of America A, 9: 1515 (1992).

[10] J. R.Fienup, "Phase retrieval algorithims: a comparison," Applied Optics, 21:2758 (1982).

[11] G. Pedrini, W. Osten, Y. Zhang, "Wave-front reconstruction from a sequence of interferograms recorded at different planes," Optics Letters, 30: 833 (2005).

[12] P. Almoro, G. Pedrini, W. Osten, "Complete Wavefront reconstruction using sequential intensity measurements of a volume speckle field," Applied Optics, 45: 8596 (2006).

[13] A. Migukin, Interative Phase Retrieval from multiple noisy observations: Variational and Sparse object approximation techniques. PhD thesis, Tampere University of Technoloy, Tampere, Finlandia (2012).

[14] J. D. Schmidt, Numerical Simulation of Optical Wave Propagation. SPIE Press, 1st edition (2010).

[15] P. Almoro, A. M. S. Maallo, S. G. Hanson, "Fast-convergent algorithm for speckle-based phase retrieval and a design for dynamic wavefront sensing," Applied Optics, 48: 1485 (2009).

[16] A. Migukin, V. Katkovnik, J. Astola, "Wave field reconstruction from multiple plane intensity-only data: augmented Lagrangian algorithm," Journal of the Optical Society of America A, 28: 993 (2011).

[17] A. Anand, G. Pedrini, W. Osten, P. Almoro, "Wavefront sensing with random amplitude mask and phase retrieval," Optics Letters, 32: 1584 (2007).

[18] A. Anand, V. K. Chhaniwal, P. Almoro, G. Pedrini, W. Osten, "Shape and deformation measurements of 3D objects using volume speckle field and phase retrieval," Optics Letters, 34: 1522 (2009)

[19] C. Falldorf, M. Algour, C. V. Kopylow, R. B. Bergmann, "Phase retrieval by means of a spatial light modulator in the Fourier domain of an imaging system," Applied Optics, 49: 1826 (2010)

[20] V. Katkovnik, J. Astola, "Phase retrieval via spatial light modulator phase modulation in $4 \mathrm{f} \mathrm{optical}$ setup: numerical inverse imaging with sparse regularization for phase and amplitude," Journal of the Optical Society of America A, 29: 105 (2012)

[21] R. A. Gonsalves, R. Chidlaw, "Wavefront sensing by phase retrieval," SPIE Applications of Digital Image Processing III, 207, 32 (1979)

[22] R. A. Gonsalves, R. Childlaw, "Phase retrieval and diversity in adaptative optics," Optical Engineering, 21: 829 (1982)

[23] R. G. Paxman, T. J. Shulz, J. R. Fienup, "Joint estimation of object and aberrations by using phase divertity," Journal of the Optical Society of America A, 9: 1072 (1992)

[24] P. Kner, "Phase diversity for three-dimensional imaging," Journal of the Optical Society of America A, 30: 1980 (2013)

[25] N. Uribe-Patarroyo, A. Álvarez Herrero, T. Belenguer, "Measurement of the quantum superposition state of an imaging ensemble of photons prepared in orbital angular momentum states using phase diversity method," Physical Review, 81: 053822 (2010)

[26] A. Jesacher, A. Schwaighofer, S. Fürhapter, C. Maurer, S. Bernet, M. Ritsch-Marte, "Wavefront correction of spatial light modulators using an optical vortex image," Optics Express, 15: 5801 (2007)

[27] J. W. Goodman, Introduction to Fourier Optics, McGraw Hill, Higher Education, $2^{\text {nd }}$ edition (1996)

[28] D. G. Voelz, Computational Fourier Optics: A MATLAB Tutorial. SPIE Press, $1^{\text {st }}$ edition (2011)

[29] R. Noll, "Zernike polynomials and atmospheric turbulence," Journal of the Optical Society of America, 66: 207 (1976)

[30] S. Echeverri-Chacón, R. Restrepo, C. Cuartas-Vélez, N. Uribe-Patarroyo. Vortex-enhanced coherentillumination phase diversity for phase retrieval in coherent imaging systems, Optics Letters, 41, 1817-1820, (2016). 


\section{Introduction}

On imaging systems, the phase that is related to the wavefront can be analyzed under two perspectives. The first one as optics phase aberrations, and second one as object phase located at the entrance plane. Iterative methods called phase retrieval methods contribute to phase recovery trough intensity observations in the image plane. Phase reconstruction has many scientific and engineering applications such as astronomy, microscopy, opto-mechanics, among others [1-4]. Normally, the phase or wavefront is measured through interferometric techniques, or others non-direct techniques i.e. Shack-Hartmann or curvature sensors, and also, phase retrieval methods. Although, the disadvantage of interferometric methods regarding to non-direct methods is the reference beam needed, recently the interferometry has had advances in this sense, eliminating it [5]. However, the phase retrieval methods offer a simple optical setup, including some configurations that allow working with partially coherent or completely incoherent light.

In 1972 Gerchgerg and Saxton (GS) [6] introduced an algorithm to determine the complete wave function, as amplitudes as phases, by recording the intensity in the image and diffracted planes. This method has been used in astronomy combined with a curvature sensor [7], and recently in human eyes aberrations studies [8]. The GS method depends on the existence of a relation of Fourier transform between its two planes, image and diffracted, and therefore, is restricted to a certain degree of spatial and/or temporal coherence of the field. In 1992, it was reported a modification of the GS algorithm, where information of multiple intensities was introduced in a series of defocused planes [9].

The methods based on GS are deterministic and they mainly solve the transport equation, those require several a priori approximations and constraints [10]. An alternative reconstruction using only one beam with multiple intensity registers (Single-beam multiple-intensity Reconstruction - SBMIR) measured on a Speckle field volume, using the wave propagation equation, was proposed in $2005[11,12]$, and this allowed that the a priori restrictions could be relaxed. These intensity registers are obtained by moving a CCD over the propagation axis, and acquiring them in different planes, then, using the RayleighSommerfeld approximation the complex amplitude is recovered. This process of multiplying the distribution of measured intensities, with the phase calculated in each plane, followed by the intensity of the wave, is repeated until reaching a quality in the reconstructed image in the object plane, with very small changes with respect to a specific error function. The numerical solution to the first approximation of the Rayleigh-Sommerfeld integral, shows similar results compared with the Angular Spectral Decomposition method (ASD) regularly used in these phase-recovering algorithms for fast convergence $[13,14]$.

The SBMIR algorithm has been improved to make faster its convergence [15], even seen from the point of view of optimization [16] and used in different applications [2, 17, 18]. However, the CCD movements represent an important issue due to mechanical misalignments, but with the Spatial Light Modulators (SLMs) development, this problem could be fixed. Especially, in a $4 \mathrm{~F}$ setup, the SLM is located in the Fourier plane and in it is projected a variable propagation transfer function for each recorded intensity [19], emulating parallel displacements of the CCD. Recently, in a 4F system and via a SLM, an iterative algorithm using random phase masks was developed with the advantages of compressive sensing and compared to the SBMIR [20].

The algorithms so far described require very high spatial and temporal coherence, the main objective of these algorithms is to recover the complex field of an extended object assuming very low aberrations in the imaging system [20]. In Astronomy, the algorithm based on GS has been used for characterization of optical aberrations using a star as object, which is a point object and has spatial coherence $[7,10]$.

Another method of phase recovery widely known in astronomy and specifically in solar physics, called Phase Diversity (PD) [1], was first proposed by Gonsalves and Childlaw [21],which infers phase aberrations by working with images of extended objects formed through an optical imaging system, illuminated with incoherent light [22].The conventional PD technique for image restoration requires the use of at least two images of the object to be reconstructed, one of which is aberrated by the optical system, and this aberration is unknown. The other image, is taken simultaneously with the first, but also has a known aberration, intentionally induced [23]. This technique has been used not only in astronomy, recently the literature reports uses in microscopy [24] and quantum optics [25]. 
The main objective of the PD method is to recover the aberrations of the system parametrized on a Zernike basis and with these in a later step of image processing to restore the acquired images to the diffraction limit [1].

The objective of the PD method is different from the aim of the methods based on GS and SBMIR. However, all methods are inverse problems, it means, that some parameters must be obtained from data coming from the observation. In the case of these methods, they are intensity measurements recorded by a detector. The methods based on GS, used for characterization of aberrations are poor compared to the PD method $[10,26]$.

This work focuses on combining the PD techniques in a $4 \mathrm{~F}$ assembly with quasi-monochromatic light, using an SLM in the Fourier plane. In this one will be projected the masks of phase diversity, which are a transmittance function of spherical character, in order to recover and compensate the system aberrations, then, using the algorithms based on GS and SBMIR (Multi-plane Phase Retrieval method), recover the object complex field, once it is illuminated with coherent light.

This paper is structured as follows: In Section 2 a mathematical formalism will be developed, Section 3 mentions some considerations about the optical setup, Section 4 shows the results obtained with the method, and finally in Section 5 the conclusions are presented

\section{Mathematical Background for imaging systems in coherent and incoherent light}

It is known that coherent systems are linear in complex fields and incoherent systems are linear in intensities [14,27,28], and there are some relationships between coherent and incoherent systems useful to describe how the phase retrieval methods works. These relationships are briefly described as follows.

Coherent systems are characterized by their impulse response $h(\vec{x})$. The convolution of the object with the impulse response that describe imaging process in coherent systems can be written in terms of a product of Fourier transforms in frequency coordinates $(\vec{u}): G(\vec{u})=G_{o b j}(\vec{u}) H(\vec{u})$, where $H(\vec{u})$ is the Fourier transform (FT) of $h(\vec{x})$ known as the generalized pupil, $G_{o b j}(\vec{u})$ represents the FT of the object complex field. The generalized pupil can be described in terms of an aperture and phase function as $H(\vec{u})=A(\vec{u}) e^{i \varphi(\vec{u})}$.

On the other hand, incoherent systems are characterized by their Point Spread Function (PSF) that is the square modulus of the impulse response $|h(\vec{x})|^{2}$. The convolution of the object intensity with the PSF that describe imaging process in incoherent systems can be written in terms of a product of Fourier transform as it was made in the coherent systems. The product is: $I(\vec{u})=I_{\text {obj }}(\vec{u}) \operatorname{OTF}(\vec{u})$, where OTF $(\vec{u})$ is the Optical Transfer Function and the FT of the PSF. The $\operatorname{OTF}(\vec{u})$ also can be defined as the normalized autocorrelation of $H(\vec{u})$.

\section{2.a Phase Diversity Method}

The Phase Diversity method is a technique that allows the estimation of $\operatorname{OTF}(\vec{u})$ in the presence of noise in $I(\vec{u})$. It consists of the measurement of nominal image $I_{1}(\vec{u})$ with its nominal system $O T F_{1}(\vec{u})$ and $K$ additional images $I_{2, \ldots, K}(\vec{u})$ in which the $O T F(\vec{u})$ of the system is changed by known amounts, therefore, the generalized pupil changes these known amount. These changes are called diversities that produce phase-diverse states. There are $\operatorname{KOTF}_{2, \ldots, K}(\vec{u})$ with known relationship to $O T F_{1}(\vec{u})$. An estimation of the nominal $\operatorname{OTF}_{1}(\vec{u})$ and of the object intensity is found by minimizing an error metric [23]. Now the agreement on the optical system aberrations $O T F_{1}(\vec{u})$ among $K$ different phase-diversity images can be expressed as a function

$$
\mathcal{L}_{M}(\tilde{a})=\sum_{\forall \vec{u} \neq 0} \frac{\left|\sum_{l=1}^{K} I_{l}(\vec{u}) O T F_{l}^{*}(\vec{u})\right|^{2}}{\sum_{l=1}^{K}\left|O T F_{l}(\vec{u})\right|^{2}}-\sum_{\vec{u}} \sum_{l=1}^{K}\left|I_{l}(\vec{u})\right|^{2},
$$

where, $\tilde{a}$ are the weights vector of a parametrized phase $(\varphi(\vec{u}))$ in a Zernike basis. Through a gradient search algorithm, the weights vector is varied until reaching convergence following Eq. (1). 
The Phase Diversity method is mainly oriented to image restoration, and its main goal is to recover the system aberrations, and then, getting the resolution limit of the observed object intensity. This method is commonly used in solar physics to identify new structures on the sun.

\section{2.b Multi-plane Phase Retrieval Method}

The Multi-plane Phase Retrieval method is a technique to recover the phase of a coherent wave field from intensity measurements, which is an inverse problem, where both amplitude and phase will be reconstructed from the data coming from the observations using a $4 \mathrm{~F}$ setup. As the Phase Diversity method, Multi-plane Phase Retrieval method assumes that registered multiple wave fields are specially modulated and those modulations are the diversities, therefore, if the system is limited by diffraction the pure object complex field is recovered. Defocusing is the most used idea to modulate the phase [13, 19, 20]. In this method, phase as well as amplitude of the wave field are reconstructed from noisy multipleintensity observations. The reconstruction is optimal due to a constrained maximum likelihood formulation of the problem. The proposed algorithm is iterative with decoupling of the inverse of the forward propagation of the wave field and the filtering of phase and amplitude [20]. The input variables are the intensity observations $o_{l}(\vec{x})$, the phase masks projected o the spatial light modulator $S L M_{l}(\vec{u})$ and the initial guess $g_{o b j}^{i n i t}(\vec{x})$ for the complex-valued object distribution $g_{o b j}(\vec{x})$. The objective function to be minimized is given by

$$
\begin{aligned}
& \mathcal{L}\left(g_{o b j}^{t},\left\{g^{t+1}\right\}_{l},\left|g_{o b j}^{t+1}\right|, \phi_{o b j}^{t+1}\right)=\sum_{l=1}^{K} \frac{1}{2 \sigma_{l}^{2}} \sum_{\vec{x}}\left(o_{l}(\vec{x})-\left|\left\{g^{t+1}(\vec{x})\right\}_{l}\right|^{2}\right)^{2}+ \\
& \sum_{l=1}^{K} \frac{1}{\gamma_{l}} \sum_{\vec{x}}\left|\left\{g^{t+1}(\vec{x})\right\}_{l}-\mathcal{F}^{-1}\left\{G_{o b j}^{t}(\vec{u}) S L M_{l}(\vec{u})\right\}\right|^{2}+ \\
& \frac{1}{\gamma_{o}}\left\|g_{o b j}^{t}(\vec{x})-\left\{\left|g_{o b j}(\vec{x})\right| e^{i \phi_{o b j}(\vec{x})}\right\}^{t+1}\right\|_{2}^{2},
\end{aligned}
$$

where, the first line of Eq. (2) is the quadratic fidelity obtained from Gaussian likelihood function and is mainly the error metric. The second and third lines are the constrains given using the quadratic penalization functions. $\sigma_{l}^{2}$ is a Gaussian noise, $\gamma_{l}$ and $\gamma_{o}$ are penalization parameters. The upper index $t$ stands for the iteration number.

The wave fields have three different forms in the algorithm and the authors exploit this fact. In spatial domain, in the frequency domain and a vectorized object image variable.

The algorithm has 3 mainly steps for no filtering version. Step 1) Using Fourier transforms, the information in the image plane for each diversity is predicted. Step 2) For each diversity the information the new iteration over the image plane is estimated by minimizing with respect the information in the old iteration over the same plane. Step 3) the minimization is respect the information estimated in the frequency domain object plane.

\section{Optical setup considerations}

Using the commercial software Zemax, the proposed $4 \mathrm{~F}$ optical system was evaluated, where the SLM is located in the Fourier plane, this can be seen in Fig.1(a). The lenses used are commercially produced by Newport, reference PAC052, these are achromatic doublets of $24.4 \mathrm{~mm}$ diameter and effective focal length (EFL) $100 \mathrm{~mm}$. The glass of the first part of the doublet is N-BK7 and the second is N-SF5. The object used to simulate the experimental setup is two-dimensional, and it is a transmittance function, represented by the letter F and shown in Fig.1(b). The wavelength chosen for the simulation was 632.8nm.

The 4F optical system used in this work shows to be advantageous due to the Fourier plane is located in the same position of the pupil. That means, the defocuses introduced for both methods explained in Section 2 are the diversities without additional distortions. On the other hand, $4 \mathrm{~F}$ optical system is a restriction for the Multi-plane Phase Retrieval method. 


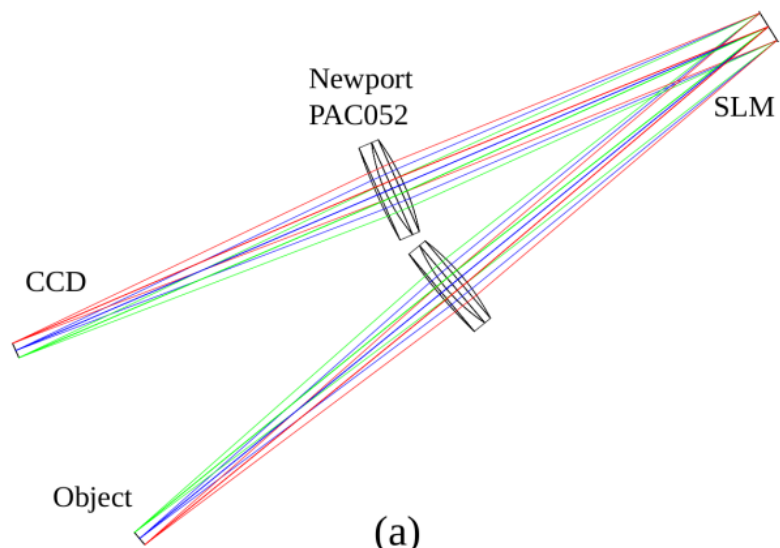

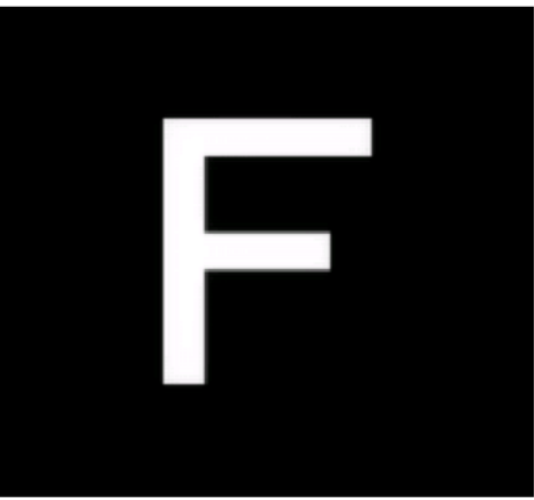

(b)

Fig. 1. (a)Setup for three different object fields. (b) Object image limited by diffraction.

In the position where the SLM is located, defocus aberrations are introduced using a Zemax function that simulates Zernike polynomials with a single index Noll notation [29], for this case, defocus is the 4th polynomial. For one wavelength of aberration, the mask in radian projected on the SLM is shown in Fig.2.

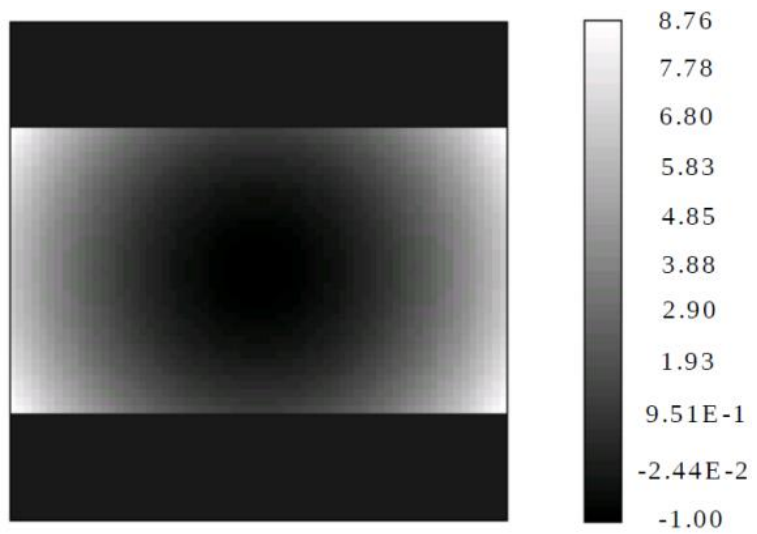

Fig. 2. One wavelength of defocus aberration projected on the SML, the units are in cycles of $2 \pi \mathrm{rad}$.

The defocuses that were used in experimental setups, for both the Phase Diversity method and the Multiplane Phase Retrieval method have the same shape as those used in this simulation.

Fig.3(a) shows the quality of the optical system through the Modulation Transfer Function (MTF), as it can be seen is very close to the diffraction limit, which indicates that the aberrations produced by the optical system are low. However, in an experimental system, aberrations may not be as low due to system misalignments, to deformations on the modulator surface or to the lenses themselves.

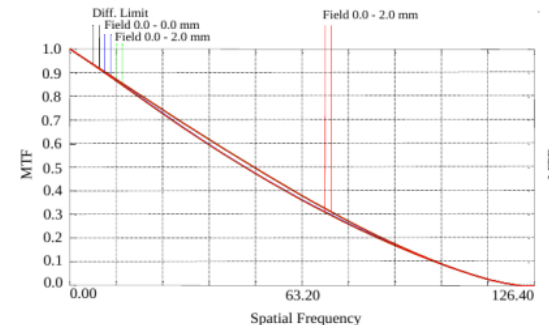

(a)

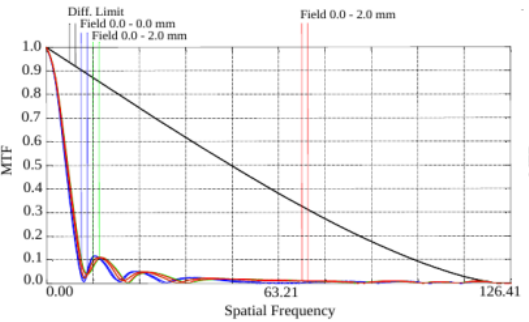

(b)

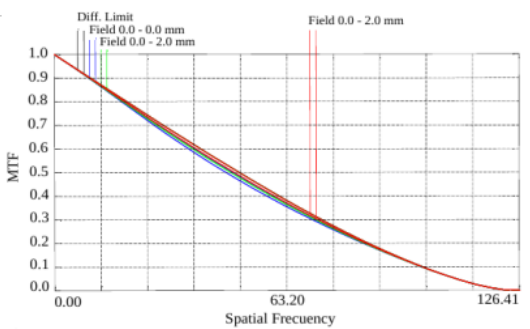

(c)

Fig 3. System MTF (a) without aberration. (b) One wavelength of defocus aberration. (c) MTF Recovered moving the CCD

Once the defocus aberration of a wavelength in the Fourier plane has been introduced, using the aforementioned 4th polynomial, there is a dramatic degradation of the MTF function as shown in Fig.3(b). Using the Zemax optimization tool, leaving the position of the CCD (Image Plane) as a free parameter, it 
can be seen that a small displacement of the CCD successfully recovers the optical quality of the system, as shown in Fig.3(c), and where MTF is recovered with the same shape before the induced aberration.

Three images of the object, with different aberrations introduced in the SLM, of one, two and four wavelengths of aberration, can be seen in Fig.4. In Table 1, different values obtained from the CCD movements are shown in order to recover the initial MTF, once aberrations of different value are introduced. When the aberration value is greater than six wavelengths, the MTF of the initial optical system can no longer be successfully recovered. According to the table, it is observed that incremental $\Delta \mathrm{z}$ with respect to the defocus introduced in the SLM, corresponds to a linear function.

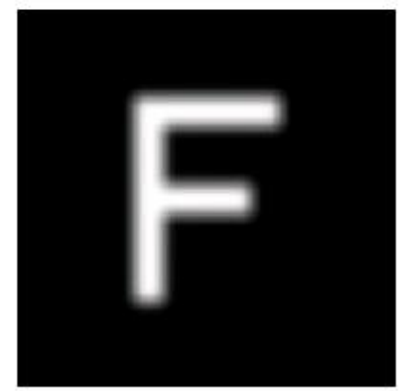

(a)

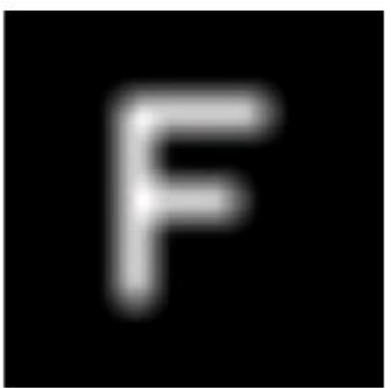

(b)

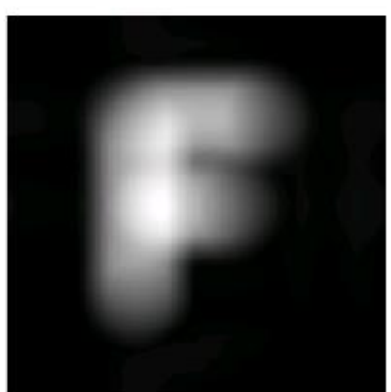

(c)

Fig. 4. Aberrated image with: (a) one wavelength. (b) Two wavelengths. (c) Four wavelengths of defocuses

Table 1. Defocus comparison on the Fourier plane regarding to the displacements of the CCD (Image plane).

\begin{tabular}{|c|c|c|c|}
\hline \hline $\begin{array}{c}\text { SLM Defocuses } \\
\text { (wavelengths) }\end{array}$ & $\Delta \mathrm{z} C \mathrm{CD}(\mathrm{mm})$ & Accumulated $\Delta \mathrm{z}(\mathrm{mm})$ & Nominal CCD $\mathrm{z}(\mathrm{mm})$ \\
\hline \hline 0 & 0 & 0 & 93.355 \\
1 & 1.594 & 1.594 & 97.949 \\
2 & 1.594 & 3.188 & 99.543 \\
3 & 1.594 & 4.781 & 101.136 \\
4 & 1.594 & 6.375 & 102.729 \\
5 & 1.594 & 7.968 & 104.323 \\
6 & 1.594 & 9.560 & 105.915 \\
\hline
\end{tabular}

\section{Results}

In this section, we present the simulated and experimental results. With the results obtained through simulations we did an analysis of the methodology proposed in the previous sections. The Phase Diversity method is used to characterize system aberrations, and the Multi-plane Phase Retrieval method is used to recover the phase of the object complex field. However, the object complex field could be aberrated due to the fact that this one is recovered by the observations on the image plane and it is common that all optical systems tend to have slight aberrations, therefore, a last step is necessary to compensate the aberrations of the system and determine the free-aberrations object complex field.

\section{4.a. Simulations}

\section{4.a.a. Object complex field and optical system}

The principal goal of the simulations is to prove how the method works before implementing it in an experimental application, which means, we have to generate a complex object, and an image on a parallel plane will be created by following the structure of a $4 \mathrm{~F}$ optical system as it was presented on Section 3. On parallel plane only intensities are detected, and their distributions depend on the light source used. Assuming a quasi-monochromatic source and low spatial coherence (incoherent light), the object is illuminated following the mathematical development explained in Section 2, and then using the Phase Diversity method (Section 2.a), the system aberrations are recovered.

Once the aberrations are recovered, and assuming a distribution of intensities on the image plane with coherent illumination (Section 2), the Multi-plane Phase Retrieval method proposed in Section 2.b is used 
to recover the phase of the object. The optical system phase recovered previously (optical aberrations) is an entry for this algorithm.

Fig.5 shows a simulated object where normalized amplitude corresponds to three bars with different transmittance value (Fig.5(a)), values from left to right are: $1 ; 0.667$ and 0.333 . The phase corresponds to three bars each one with different value of the phase (Fig.5(b)), the values of the phase were taken arbitrarily and correspond from left to right: $1.15 \mathrm{rad}, 2.7 \mathrm{rad}$ and $-2 \mathrm{rad}$.

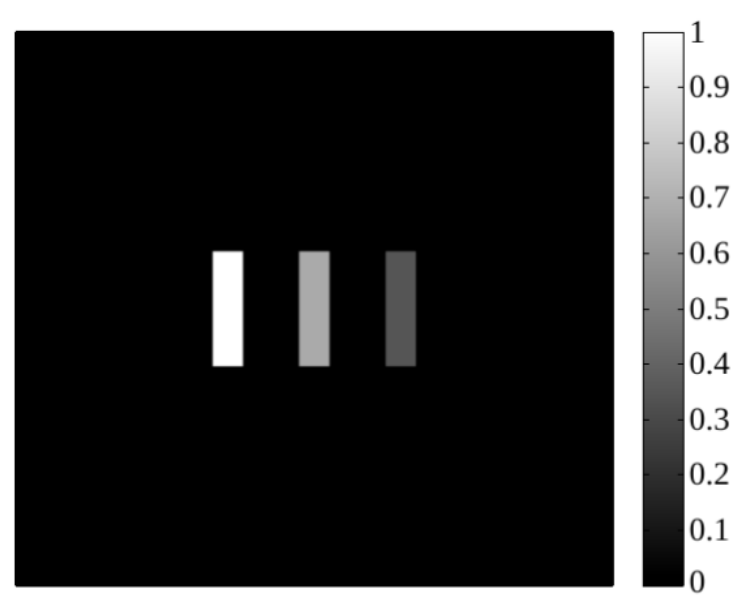

(a)

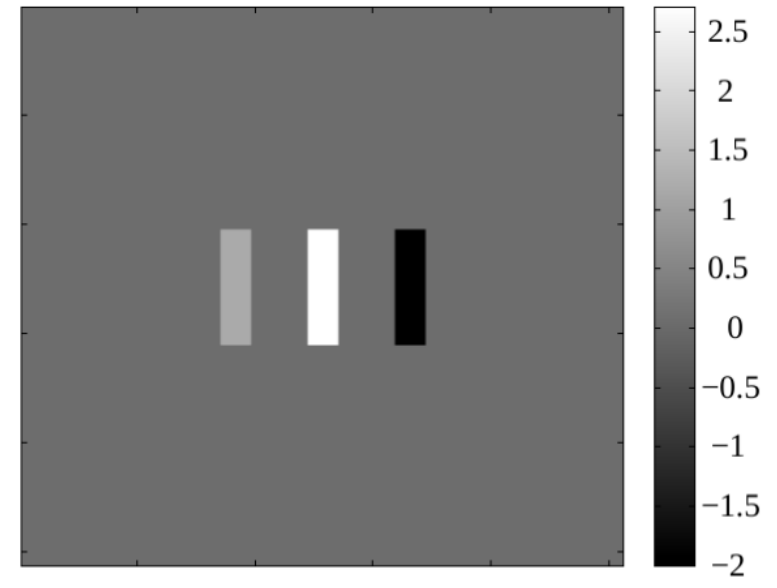

(b)

Fig. 5. Simulated object complex field. (a) Amplitude (the amplitude values correspond from left to right a: $1 ; 0.667$ and 0.333 ). (b) Phase (Phase values correspond from left to right a: $1.15 \mathrm{rad}, 2.7 \mathrm{rad}$ and $-2 \mathrm{rad}$ ).

If the object is seen though an optical system without aberrations, that means, the generalized pupil has a constant unit value transmittance in the amplitude, and the wavefront is plane with zero phase value, the object amplitude has a decay of the intensity, and diffraction structures are appreciated in the phase. In the example (Fig.6), the diffraction is amplified by the low numerical aperture simulated. In general, the optical systems seen from signal theory are pass-low filters.

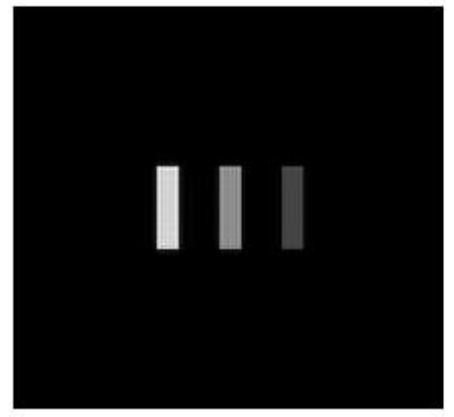

(a)

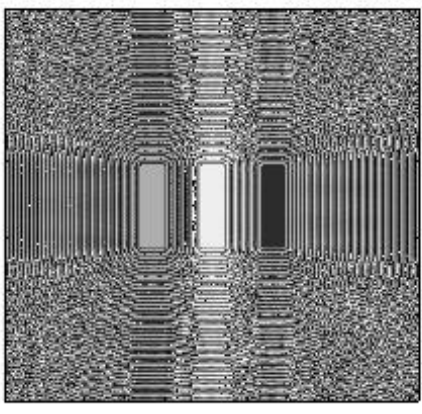

(b)

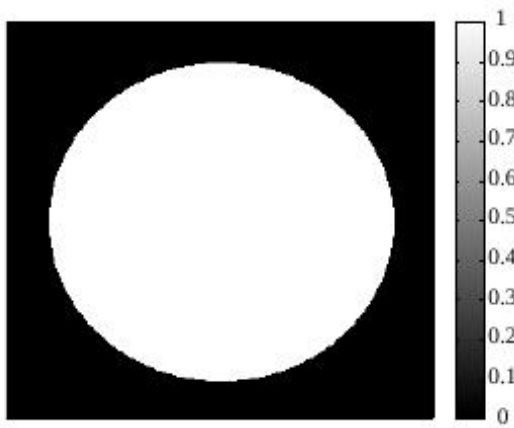

(c)

Fig. 6. Object seen from the image plane with free-aberrations optic system. (a) Normalized amplitude. (b) Modulo $2 \pi$ phase. (c) Generalized pupil amplitude.

On the other hand, if an aberration is introduced to the system through a generalized pupil as it is described on Section 2, both the amplitude and the object phase will be deformed. Fig.7 shows the effects.

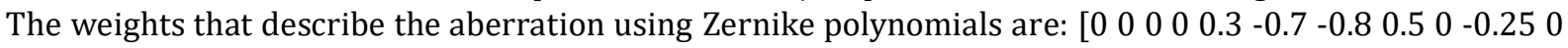
$\left.\begin{array}{llll}0 & 0 & 0 & 0\end{array}\right]$.

\section{4.a.b. Phase Diversity Method}

In this subsection, the results obtained by Phase Diversity method are shown when the simulated optical system has the aberrations described in the last paragraph in a Zernike weights decomposition. The 
difference between the expected weights by Phase Diversity method and the weights described in the last paragraph must approach to zero.

If the aberrated system is illuminated with incoherent light, then, defocuses are introduced as it was explained in Section 2.a, and additionally, Phase Diversity method is applied, using 8 defocuses of $0.25 \lambda$ each one, the intensity of the object without aberrations in the image plane and the aberrations of the system are recovered. The error RMSE was 0.0102 , compared with the weights induced. The recovered

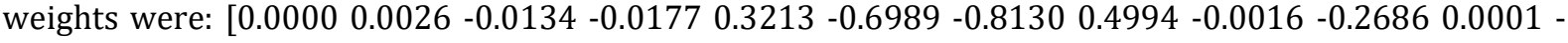
$0.00050 .00070 .0085-0.0007]$.

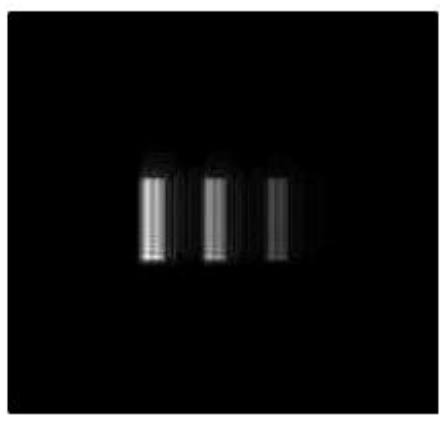

(a)

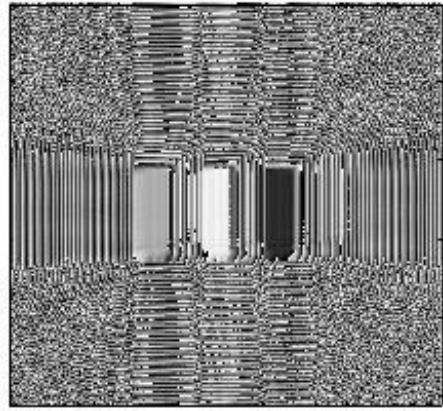

(b)

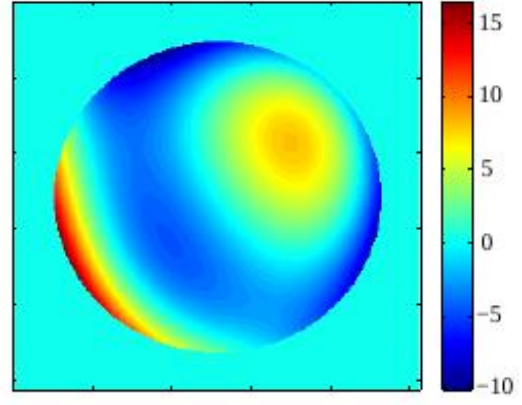

(c)

Fig. 7. Object seen from the image plane with aberrated optic system. (a) Normalized amplitude. (b) Modulo $2 \pi$ phase. (c) Generalized pupil (wavefront in radians).

\section{4.a.c. Multi-plane Phase Retrieval Method}

Using the Multi-plane Phase Retrieval method to recover the object complex field described in Section 2.b, with the same defocuses used by the Phase Diversity method, the amplitude of the object with the effects of the aberrations is shown in Fig.8(a) and the phase in Fig.8(b).

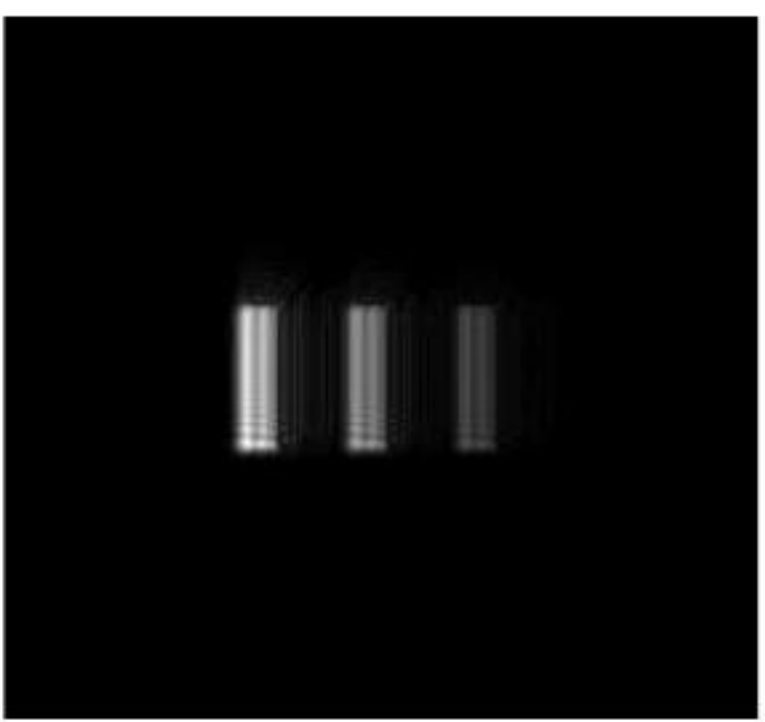

(a)

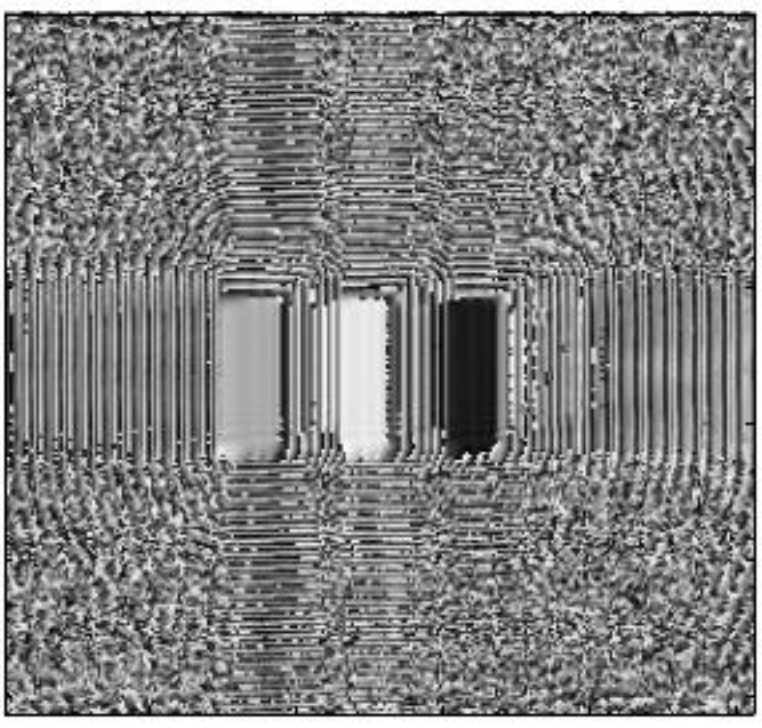

(b)

Fig. 8. Object complex field recovered with aberrated optical system seen from the image plane, using the Multi-plane Phase Retrieval method. (a) Normalized amplitude. (b) Modulo $2 \pi$ phase.

Note from Fig.8(b) that the object phase is deformed because the information on the image plane is a combination between the filtered object phase and the aberrations of the system. The main goal of the work is to propose a feedback methodology to find the aberrations of the system and then proving 
through other different way that they represent the optical system. Therefore, if the aberrations found by Phase Diversity method are compensated and then the Multi-plane Phase Retrieval method is used, the free-aberrations object complex field is recovered. In a $4 \mathrm{~F}$ system compensating the aberrations is an easy task because the generalized pupil coincides with the Fourier plane (see Section 3). It means, the Fourier transform of the object complex field seen from image plane is multiplied with the negative of the aberrated generalized pupil, then, a new Fourier transform is performed, obtaining the object complex field seen from the image plane with the aberrations compensated. This can be deduced from the second line in the Eq. (2), and the effect can be seen in Fig.9.

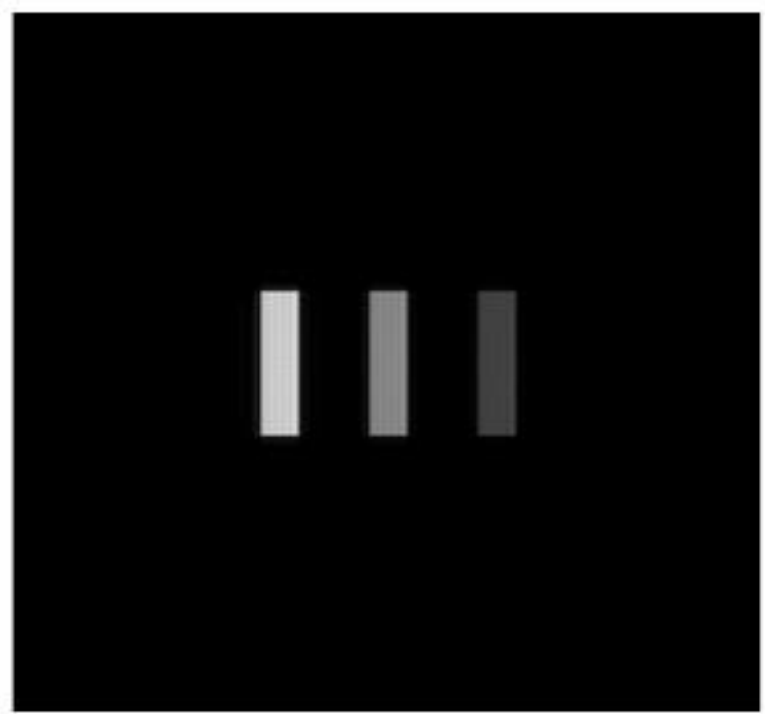

(a)

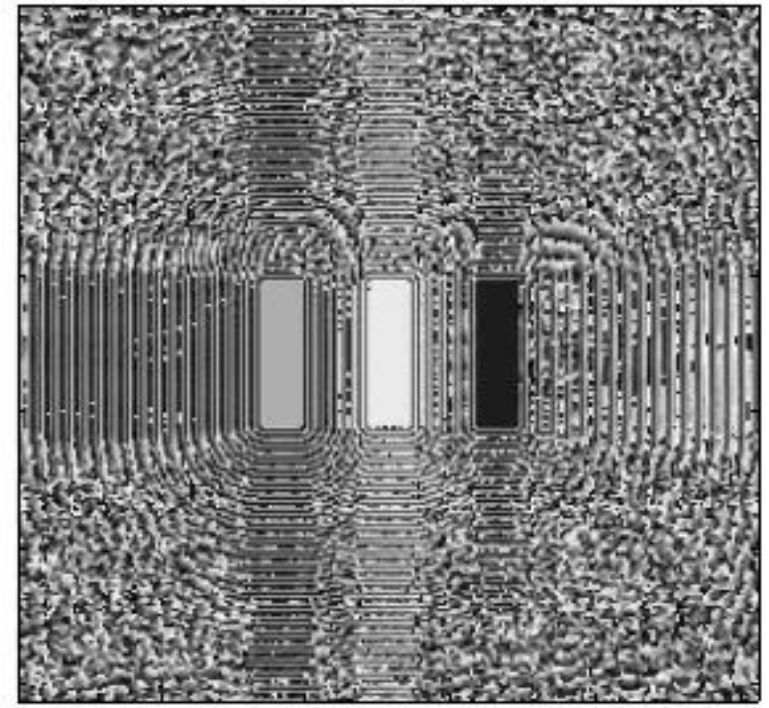

(b)

Fig. 9. Object Complex field recovered with aberrations system compensated. (a) Normalized amplitude. (b) Modulo $2 \pi$ phase.

The results about the Multi-plane Phase Retrieval method are summarized in Table 2, where it shows a comparison with the different RMSE errors. It can be seen in the third row, the comparison between the object complex field and this one recovered formed by an optical system without aberrations. The last row of the table shows the comparison between object complex field and this one recovered and compensated. The RMSE values are in the same order, showing the efficiency of the method. From the first row, the comparison between the object complex field seen in the object plane and in the image plane has an error higher than others, because the phase outside the region of interest, behaves like noise and increases the RMSE value.

TABLE 2. The Multi-plane Phase Retrieval method errors comparison.

\begin{tabular}{|c|c|c|}
\hline Comparison & $\begin{array}{c}\text { RMSE } \\
\text { (Phase) }\end{array}$ & $\begin{array}{c}\text { RMSE } \\
\text { (Ampl.) }\end{array}$ \\
\hline Object Complex Field vs. Image Complex Field (Not Aberrated Sys.) & 0.0066 & $2.812 \mathrm{e}-4$ \\
\hline Image Complex Field (Not Aberrated Sys.) vs. Image Complex Field (Aberrated Sys.) & 0.0020 & $2.326 \mathrm{e}-4$ \\
\hline Image Complex Field vs. Recovered Image Complex Field (Both Not Aberrated Sys.) & $4.816 \mathrm{e}-4$ & $1.404 \mathrm{e}-5$ \\
\hline Image Complex Field vs. Recovered Image Complex Field (Both Aberrated Sys.) & $4.888 \mathrm{e}-4$ & $1.861 \mathrm{e}-5$ \\
\hline Image Complex Field (Not Aberrated Sys.) vs. Recovered Image Complex Field (Aberrated Sys.) & $4.572 \mathrm{e}-4$ & $9.234 \mathrm{e}-5$ \\
\hline
\end{tabular}

\section{4.b. Experimental}

The experimental setup follows the configuration shown in Fig.1(a), the stop aperture is located in the position of the SLM (Holoeye Pluto-Vis), the elements used in the arrangement were: A commercial super bright LED and a commercial interferential bandpass filter (Newport reference 10BF10-630), centered in $630 \pm 2 \mathrm{~nm}$ and with a bandwidth of $10 \pm 2 \mathrm{~nm}$ following the criteria FWHM (Full Width at half Maximum), which produced quasi-monochromatic light. This illumination was used in the Phase Diversity Method, for 
the recovery of the aberrations system. Also a He-Ne of $632 \mathrm{~nm}$ was used to illuminate the object, this illumination was used in the Multi-plane Phase Retrieval method for recovering object phase. The object to study is a Euro French coin, shown in Fig.10.

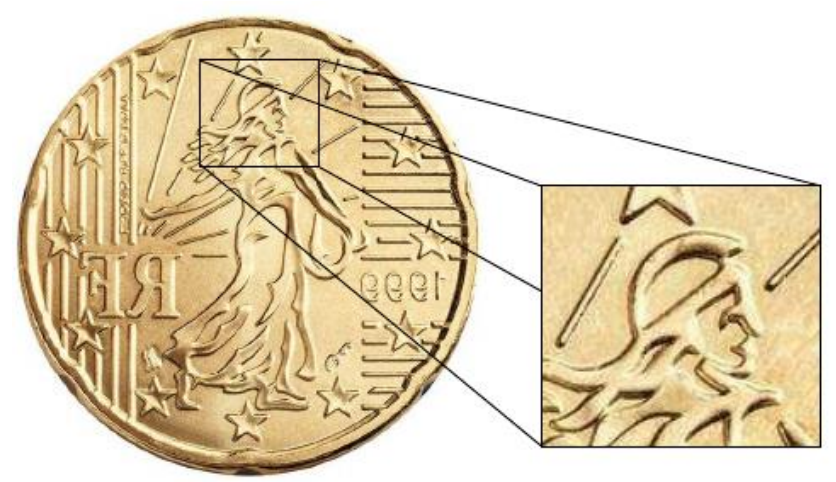

Fig. 10. 20 cents Euro coin (France 1999)

The illuminated object with both sources is shown in Fig.11. Note the effect of the Speckle on the object when is illuminated with coherent light, compared to the image of the object when is illuminated with incoherent quasi-monochromatic light.

The periodic structure of the SLM produces non appreciable diffraction effects in the experiment (Fig.11), but this structure and the location of the SLM are generating attenuation and cutting over the frequencies of the complex objet field and optical system. That means, a general decrease over the image quality. The transfer function represented by discretized free aberrations pupil is altered, therefore, the best image quality formed by the system using a mirror in the SLM location (diffraction limited) cannot reconstructed.

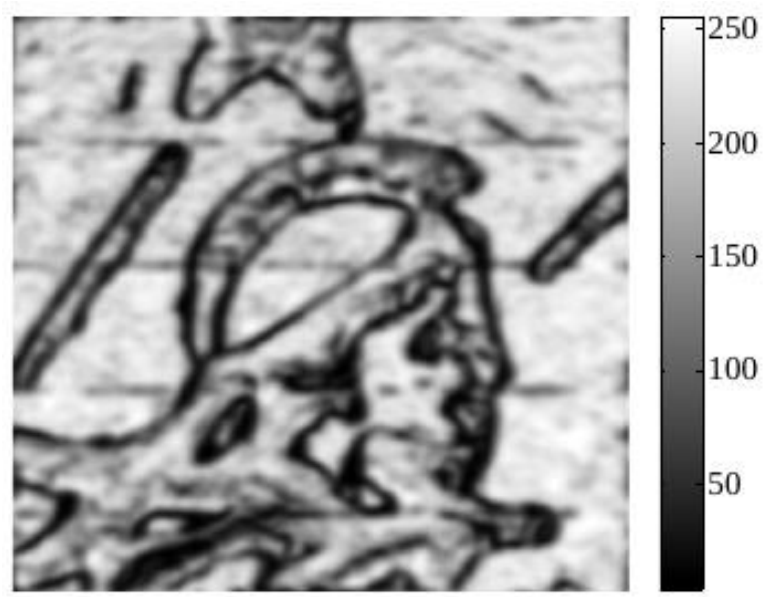

(a)

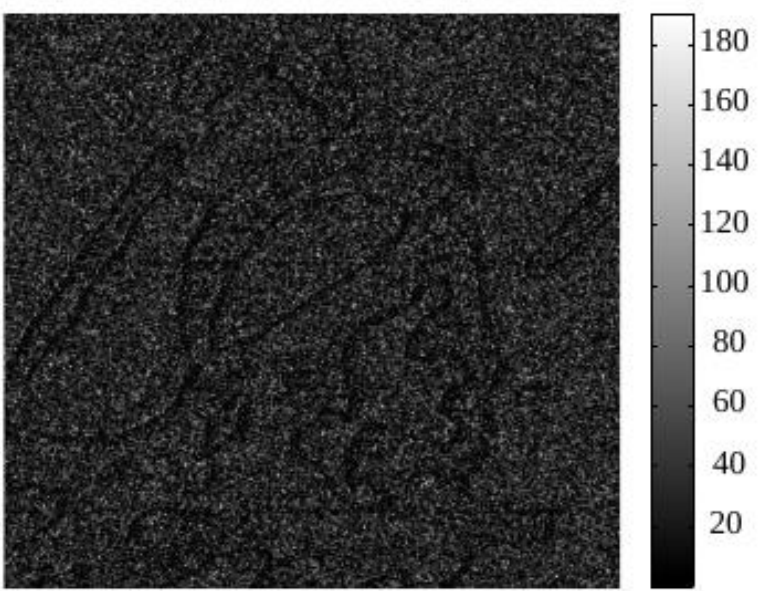

(b)

Fig. 11. Illuminated object with: (a) Incoherent Light and (b) Coherent light.

In the experiment, 8 defocuses of $0.25 \lambda$ were used following the implemented methodology in the simulation, both for the use of the Phase Diversity method and for the Multi-plane Phase Retrieval method.

The Fig.12(a) shows the intensity recovered in the image plane using the Phase Diversity method, the image sharpness, therefore its quality, is slightly better from the qualitative point of view than of the original input image (See Fig.11(a)). The Fig.12(b) shows the aberrations of the system in radians, they were found using the Phase Diversity method in a $4 \mathrm{~F}$ setup. 
Applying the Multi-plane Phase Retrieval method and compensating the aberrations obtained both the amplitude and the phase of the object are recovered. There are two ways to compensate the aberrations. The first one, is experimental, because the aberrations can be projected directly on the SLM. The second one, is in post-processing. With both ways getting the same results. Fig.13 shows the phase of the object complex field after phase unwrapping with and without aberrations compensated. It can be seen in Fig 13(b) that the free-aberrations object phase is better recovered.

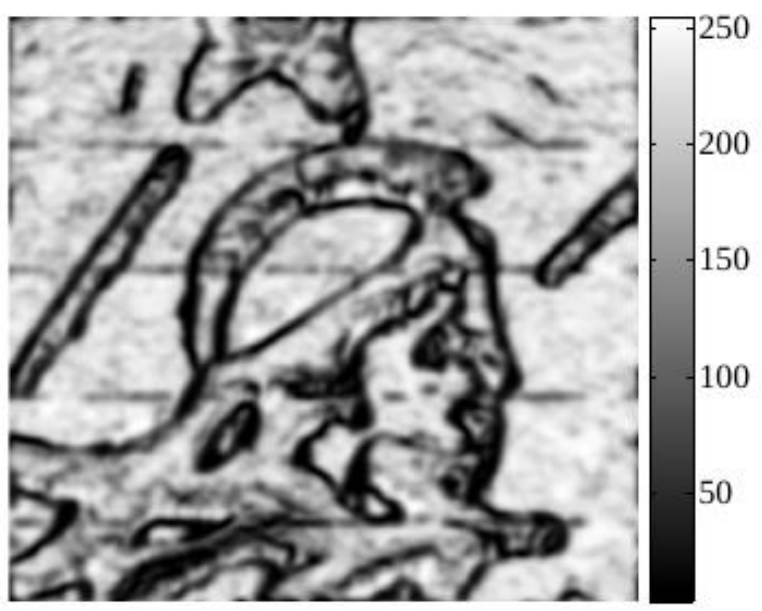

(a)

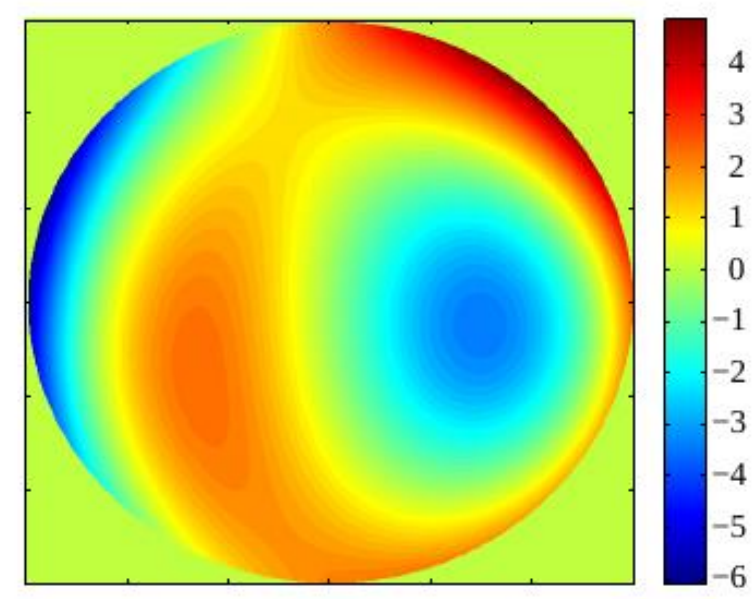

(b)

Fig. 12. Use of the Phase Diversity method for the recovery of (a) intensity in the image plane (b) and system aberrations (radians).

The height of the coin was measured with confocal microscope and the average height from the coin symbol to the semi-flat part was $40 \mu \mathrm{m}$ approximately. With the method described, the average height in radians is about 28rad approximately, which it is equivalent to $2.8 \mu \mathrm{m}$ using the working wavelength of $632.8 \mathrm{~nm}$. The difference in the order of magnitude with the results shown, is because the algorithm was modified using an arbitrary wavelength of $10 \mu \mathrm{m}$. This modification was performed because if the algorithms were used with the working wavelength, the high number of $2 \pi$ cycles would not comply the Nyquist-Shannon criterion, which means, phase shifts were not well sampled between $-\pi$ and $\pi$, therefore, the algorithm of phase retrieval did not converge. If the phase (28rad) is rescaled using the arbitrary wavelength, the phase obtained is $44.6 \mu \mathrm{m}$ of height, which coincides in magnitude with the results obtained with the confocal microscope.

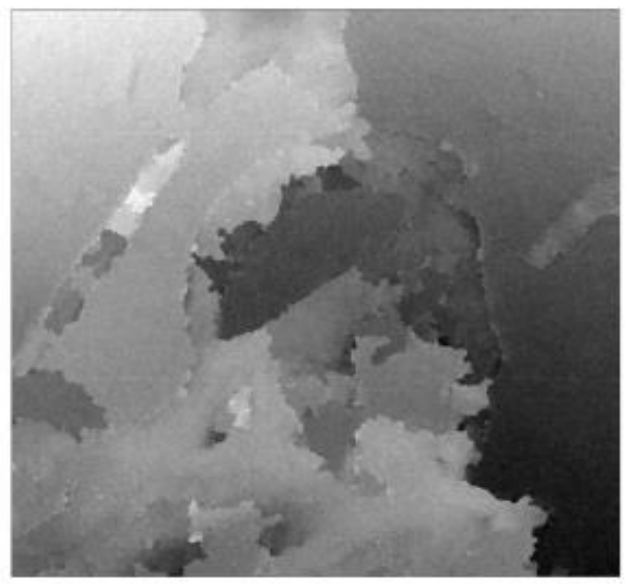

(a)

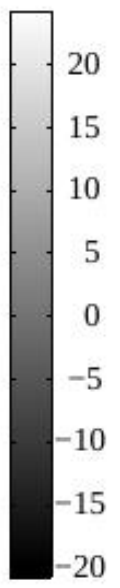

20

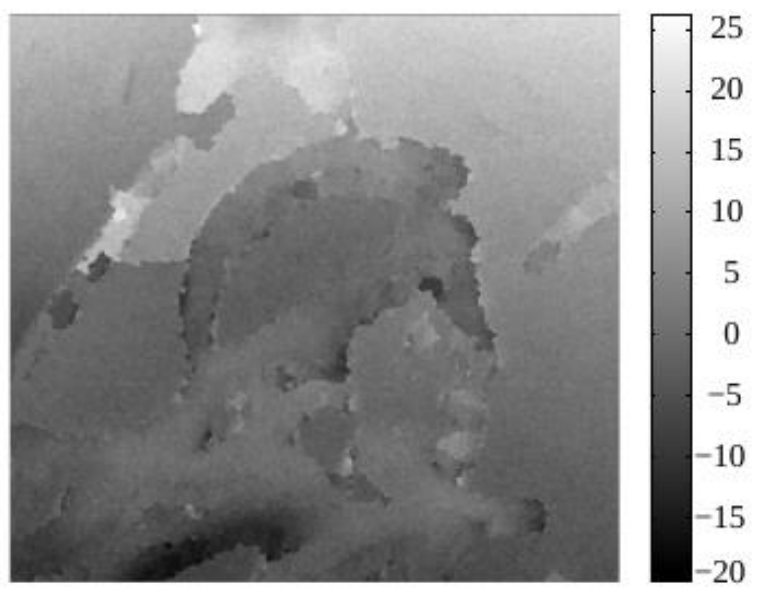

(b)

Fig. 13: Recovered phases in radians. (a) Without aberrations compensation. (b) With aberrations compensation. 
As a summary, the convergence of the method depends on three factors; the objects depth, the working wavelength and to guarantee the dimension between the numerical aperture of the experimental setup and the construction of the generalized pupil in both iterative methods.

The aerospace industry uses different techniques coming from optical metrology for the assembly and integration of instruments with optical properties. The iterative methods are other alternative for this purpose where classic techniques are not appropriated. The methodology described is a way to prove how the aberrations were correctly found, because if the object complex field is known the error of its reconstruction should be low as it was shown in the simulations. In this sense, new iterative methods have been developed with very little error for this purpose [30].

\section{Conclusions}

The Phase Diversity method and the Multi-plane Phase Retrieval method are easy to implement and use. Additionally, the mechanisms that generate the phase diversities can be replaced by SLMs, and this makes the methods more versatile. The system implemented is not only useful for the detection of aberrations, but can be corrected in the same SLM used to generate diversities.

The combination of a method which characterizes aberrations with a method which recovers the object complex field in the same configuration $4 \mathrm{~F}$ just by changing the illumination of the object, is a useful tool in the integration of optical instruments. However, the integration of optical instruments is not the only application, this implementation is also useful for the detection of phase objects in microscopy. 